\title{
LETTERS
}

\section{Bronchoscopy is useful for diagnosing smear-negative tuberculosis in HIV-infected patients}

\section{To the Editors:}

Tuberculosis (TB) is the leading cause of morbidity and mortality in HIV-infected patients in sub-Saharan Africa [1], in part because limited availability of diagnostic tests hinders early, directed treatment. Studies have demonstrated a substantial yield of bronchoscopy for diagnosing HIVassociated opportunistic pulmonary diseases, but few studies have explicitly considered whether bronchoscopy adds to the sensitivity of sputum culture in identifying Mycobacterium tuberculosis, or whether bronchoscopy shortens the time needed to diagnose TB. Although bronchoscopy is unavailable in many HIV and TB endemic settings, where it is available its usefulness for TB diagnosis is uncertain. Thus, we examined the performance of bronchoscopy to diagnose TB and other pulmonary diseases in HIV-infected inpatients with cough in Kampala, Uganda.

We performed a prospective cross-sectional study enrolling consecutive HIV-infected patients aged $\geqslant 18$ yrs hospitalised at Mulago Hospital with cough of $\geqslant 2$ weeks but $<6$ months duration. After providing informed consent, patients underwent a standard evaluation including chest radiography, sputum acid-fast bacillus (AFB) microscopy and bronchoscopy with bronchoalveolar lavage (BAL) if they were AFB smearnegative, according to previously described protocols [2]. Trained technicians examined BAL by smear and/or culture for mycobacteria, Pneumocystis jirovecii, and other fungi. Specific pneumonia treatment was recorded. Patients were seen at a 2-month follow-up visit, after which a pulmonologist and a medical officer assigned final diagnoses based on all diagnostic information and according to a standardised protocol. A final diagnosis of pulmonary TB was based on a positive sputum mycobacterial culture (using LowensteinJensen media), positive BAL AFB smear or BAL mycobacterial culture, or a clinical response to TB treatment at the 2-month follow-up visit.

Between September 2007 and July 2008, 107 (55\%) out of 193 patients successfully underwent bronchoscopy with BAL. Among the 86 patients who did not undergo bronchoscopy, 24 patients had an alternate diagnosis established, 26 refused the procedure and 20 died before bronchoscopy could be performed. Baseline demographic and clinical characteristics were similar among patients who underwent and who did not undergo bronchoscopy. Median time from enrolment to bronchoscopy was 4 days (interquartile range (IQR) 2-6 days). The median amount of saline instilled was $100 \mathrm{~mL}$ (IQR 100$125 \mathrm{~mL}$ ) with a median BAL return of $53 \mathrm{~mL}$ (IQR 50-57 mL). There were no important procedural complications arising from bronchoscopy.
$41(38 \%)$ patients received a diagnosis as a direct result of bronchoscopy. This included 18 cases of pulmonary TB (eight BAL culture-positive, 10 BAL smear-positive), 15 cases of Cryptococcus neoformans pneumonia (15 BAL culture-positive, including seven with encapsulated yeast seen on microscopy), nine cases of pulmonary Kaposi's sarcoma (KS), and five cases of Pneumocystis pneumonia (PCP). Six patients had more than one diagnosis. $28(68 \%)$ out of 41 patients received an immediate diagnosis from BAL smear or airway inspection for pulmonary KS.

A final diagnosis of pulmonary TB was made in 39 patients and definitively excluded in 39 patients. For the remaining 29 patients, 2-month follow-up information was not available (e.g. patient died) and a final diagnosis could not be made. BAL smear and culture identified the same proportion (18 out of 39; $46 \%$ ) of pulmonary TB cases as two sputum cultures (18 out of $39 ; 46 \%)$. However, these two strategies identified different but intersecting sets of individuals: eight of the 18 BAL smear- or culture-positive patients had negative sputum cultures, and eight of the 18 sputum culture-positive patients had negative BAL smears and cultures. The sensitivity of sputum culture $(46 \%, 95 \%$ CI $30-63 \%)$ or BAL culture $(41 \%$, 95\% CI $26-58 \%$ ) for TB tended to be higher than the sensitivity of BAL smear $(26 \%, 95 \%$ CI $13-42 \% ; p=0.074$ and $p=0.11$ for the pair-wise comparisons, respectively).

The median time from enrolment to TB diagnosis was 5.5 days (IQR 3-7 days) for BAL smear, compared with 22 days (IQR $8.5-34.5$ days $)$ for sputum or BAL culture $(p<0.001)$. In addition, BAL smear diagnosed a second infection in two TB patients (Cryptococcus in one, PCP in another). Four out of 10 TB patients with normal chest radiographs were diagnosed with TB by bronchoscopy alone.

Among patients with negative sputum smears and cultures, BAL smear and culture identified eight additional cases of $\mathrm{TB}$ increasing the sensitivity of diagnostic evaluation from 46 to $67 \%$ (incremental sensitivity $21 \%, 95 \%$ CI 9-36\%) (table 1). Of the eight additional TB cases, six were diagnosed by BAL smear (incremental sensitivity 15\%, 95\% CI 6-30\%) and two by BAL culture alone (incremental sensitivity 5\%, 95\% CI 1-17\%).

This study demonstrates that fiberoptic bronchoscopy is useful in HIV-infected, AFB sputum smear-negative patients with persistent cough in Uganda. Bronchoscopy confirmed a final diagnosis in $38 \%$ of patients undergoing the procedure, including TB, cryptococccal pneumonia, pulmonary KS and PCP. It increased the diagnostic yield for pulmonary TB and decreased the time required for a microbiologically confirmed diagnosis of TB. These findings are important for clinicians caring for HIV-infected patients in settings with access to 
TABLE 1 The contribution of sputum and bronchoalveolar lavage (BAL) to the diagnosis of smear-negative tuberculosis (TB)

\begin{tabular}{|c|c|c|c|c|}
\hline Specimen and test & $\begin{array}{l}\text { Test positives } \\
\text { with } \mathrm{TB}^{\#}\end{array}$ & $\begin{array}{l}\text { Additional test } \\
\text { positives with TB }\end{array}$ & $\begin{array}{l}\text { Sensitivity } \\
(95 \% \mathrm{Cl})\end{array}$ & $\begin{array}{l}\text { Incremental sensitivity } \\
\qquad(95 \% \mathrm{Cl})\end{array}$ \\
\hline Sputum culture & 18 & 18 & $46(30-63)$ & $46(30-63)$ \\
\hline BAL culture or BAL smear or sputum culture & 26 & 2 & $67(50-81)$ & $5(1-17)$ \\
\hline $\begin{array}{l}\text { BAL culture or BAL smear or sputum culture } \\
\text { or TB treatment response }\end{array}$ & 39 & 13 & $100(91-100)$ & $33(16-51)$ \\
\hline
\end{tabular}

Cultures of sputum collected on day 1 (spot collection) and day 2 (early morning collection). ${ }^{\#}: \mathrm{n}=39$; ${ }^{\circ}$ : incremental sensitivities may not sum exactly due to rounding

bronchoscopy and provide a clear rationale for its use in these settings. These findings are also important for clinical investigators choosing reference standards for studies of novel TB diagnostics in countries with high prevalences of HIV and TB.

Our findings are consistent with previous studies demonstrating a high yield of bronchoscopy in HIV-infected TB suspects in high-burden countries [3]. In the current study, bronchoscopy with BAL diagnosed about half of all TB cases, and in one-quarter of all TB cases, BAL smear reduced average time to a microbiologically confirmed diagnosis by up to 3 weeks. Earlier diagnosis is shown to have important implications both for improved individual patient outcomes and for tuberculosis infection control [4], particularly in areas where TB-drug resistance and HIV infection are frequent [5].

Although bronchoscopy substantially increased the numbers of patients diagnosed with pulmonary TB and other pulmonary diseases, it is equally notable that in $27 \%$ of patients who underwent bronchoscopy and BAL it was not possible to ascertain whether they had TB or not. Sputum culture detected less than half of all TB cases in our study, and one-third of all TB diagnoses was based on a clinical response to treatment alone. These findings emphasise the need for more sensitive diagnostic tests for TB in HIV-infected patients.

In our study, we found bronchoscopy safe, without significant complications. However, bronchoscopy is expensive and the requisite personnel and equipment are not widely available in countries like Uganda. This may limit the generalisability of these results. As an alternative approach, some studies suggest that induced sputum greatly enhances the diagnosis of TB [6] and that the yield of serial induced sputa for TB diagnosis may be similar to or better than that of bronchoscopy $[7,8]$. Because sputum induction has a lower sensitivity than BAL for PCP and other pulmonary diseases diagnosed by bronchoscopy, this should be further examined in prospective studies.

This study also provides important information about the limitations of sputum culture on solid media as a TB reference standard, especially in HIV-infected patients. Sputum culture contributed to the diagnosis of only $46 \%$ of $\mathrm{TB}$ and the use of bronchoscopy increased the number of microbiologically confirmed cases from 18 to 26 , a relative increase of $44 \%$. In a TB diagnostics study, these differences would lead to substantial increases in study power and substantial decreases in the number of patients with indeterminate or misclassified diagnoses. A recent study investigating the role of augmented methods of sputum collection in diagnosis of TB in HIV-infected patients found that liquid culture on three sputum samples detected TB in $84 \%$ compared with $62 \%$ by solid culture methods [9]. This further emphasises the need to improve the gold standard for TB diagnosis in HIV-infected populations.

There are some limitations to our study. First, $45 \%$ of patients eligible for bronchoscopy did not undergo the procedure. Onequarter of these died before bronchoscopy, a statistic which reflects the severity of illness among hospitalised patients with pneumonia. Secondly, we performed mycobacterial cultures only on solid media and not in liquid media. Liquid media detects $\sim 10 \%$ more TB cases than solid media, and requires a shorter incubation period for mycobacterial growth [10], performance characteristics which might have reduced the time and sensitivity benefits that we observed when comparing bronchoscopy to sputum cultures. While the decrease in time-todiagnosis of TB reported for liquid culture compared with solid culture is substantial, it is less than the 16-day decrease which we observed for BAL smear compared to solid culture [10].

In conclusion, bronchoscopy with BAL improves the speed and increases the sensitivity of TB diagnosis in HIV-infected, AFB sputum smear-negative inpatients with cough and where available should be considered complementary to sputum cultures in the evaluation of TB suspects in countries where HIV / AIDS is common. Future studies of the overall yield and cost-effectiveness of bronchoscopy compared to sputum induction would help to determine the best strategy for routine evaluation of HIV-infected smear-negative TB suspects in resource-limited countries.

\section{W. Worodria ${ }^{*, \#, f}$, J.L. Davis ${ }^{\#, \Phi,+, f}$, A. Cattamanchi ${ }^{\#, \Phi,+}$, A. Andama*,\#, S. den Boon ${ }^{\#}$, S.D. Yoo ${ }^{*, \#}$, P.C. Hopewell ${ }^{+}$and L. Huang $\#, \oplus, \S$}

*Dept of Medicine, Mulago Hospital, Makerere University, and "MU-UCSF Research Collaboration, Kampala, Uganda. "Division of Pulmonary and Critical Care Medicine, Dept of Medicine, San Francisco General Hospital, University of California, San Francisco, ${ }^{+}$Francis J. Curry National Tuberculosis Center, San Francisco, ${ }^{5}$ HIV/AIDS Division, Dept of Medicine, San Francisco General Hospital, University of California, San Francisco, CA, USA. ${ }^{f}$ Both authors contributed equally to the study. 
Correspondence: W. Worodria, Dept of Medicine, Mulago Hospital, Makerere University, Kampala, Uganda; MU-UCSF Research Collaboration, Kampala, Uganda. E-mail: worodria@ yahoo.com

Support Statement: This work was financially supported by the National Institutes of Health (K23 HL094141 (A. Cattamanchi), K23 AI080147 (J.L. Davis), K24 HL087713 (L. Huang), R01 HL090335 (L. Huang)); and the National Center for Research Resources (KL2 RR024130).

\section{Statement of Interest: None declared.}

Acknowledgements: This work was performed at Mulago National Referral Hospital in Kampala, Uganda, and the authors wish to thank the patients who participated in this study, the Mulago Hospital staff and administration, and the laboratory technicians at the Uganda National Tuberculosis Reference Laboratory.

\section{REFERENCES}

1 Corbett EL, Watt CJ, Walker N, et al. The growing burden of tuberculosis: global trends and interactions with the HIV epidemic. Arch Intern Med 2003; 163: 1009-1021.

2 Cattamanchi A, Davis JL, Worodria W, et al. Sensitivity and specificity of fluorescence microscopy for diagnosing pulmonary tuberculosis in a high HIV prevalence setting. Int J Tuberc Lung Dis 2009; 13: 1130-1136.

3 Velez L, Correa LT, Maya MA, et al. Diagnostic accuracy of bronchoalveolar lavage samples in immunosuppressed patients with suspected pneumonia: analysis of a protocol. Respir Med 2007; 101: 2160-2167.

4 Greenaway C, Menzies D, Fanning A, et al. Delay in diagnosis among hospitalized patients with active tuberculosis-predictors and outcomes. Am J Respir Crit Care Med 2002; 165: 927-933.

5 Gandhi NR, Moll A, Sturm AW, et al. Extensively drug-resistant tuberculosis as a cause of death in patients co-infected with tuberculosis and HIV in a rural area of South Africa. Lancet 2006; 368: $1575-1580$

$6 \mathrm{Al}$ Zahrani K, Al Jahdali H, Poirier L, et al. Yield of smear, culture and amplification tests from repeated sputum induction for the diagnosis of pulmonary tuberculosis. Int J Tuberc Lung Dis 2001; 5 . 855-860.

7 Ganguly KC, Hiron MM, Mridha ZU, et al. Comparison of sputum induction with broncho-alveolar lavage in the diagnosis of smearnegative pulmonary tuberculosis. Mymensingh Med J 2008; 17: 115-123.

8 Conde MB, Soares SL, Mello FC, et al. Comparison of sputum induction with fiberoptic bronchoscopy in the diagnosis of tuberculosis: experience at an acquired immune deficiency syndrome reference center in Rio de Janeiro, Brazil. Am J Respir Crit Care Med 2000; 162: 2238-2240.

9 Monkongdee P, McCarthy KD, Cain KP, et al. Yield of acid-fast smear and mycobacterial culture for tuberculosis diagnosis in people with human immunodeficiency virus. Am J Respir Crit Care Med 2009; 180: 903-908.

10 Chien HP, Yu MC, Wu MH, et al. Comparison of the BACTEC MGIT 960 with Lowenstein-Jensen medium for recovery of mycobacteria from clinical specimens. Int J Tuberc Lung Dis 2000; 4: 866-870.

\section{Sputum induction versus gastric washing for the diagnosis of pulmonary mycobacterial disease}

\section{To the Editors:}

The diagnosis of pulmonary mycobacterial disease is based primarily on the isolation of mycobacteria in sputum specimens. The collection of at least three samples of spontaneously expectorated sputum on three different days is the standard diagnostic procedure for patients with suspected pulmonary tuberculosis and non-tuberculous mycobacterial lung disease $[1,2]$. For patients who are unable to expectorate, bronchial secretions, aspirated by fiberoptic bronchoscopy, or gastric washings (GWs) are collected [1,3]. These procedures require the patient to be hospitalised, are fastidious and unpleasant for the patient, and the yield of GWs for the diagnosis of pulmonary tuberculosis is considered to be rather low [3].

Another option is sputum induction (SI) with nebulised hypertonic saline $[4,5]$. SI does not necessitate the patient to be hospitalised. However, it is a complicated procedure that requires trained staff and respiratory isolation conditions [4].
The diagnostic value of this procedure is still debated: several studies have reported that sputum induction has a rather low diagnostic yield [6]; other studies have found that SI compares favourably with GW and bronchoalveolar lavage in patients with suspected pulmonary tuberculosis [4]. We therefore decided to conduct a prospective study to compare the diagnostic value of SI with that of GW in adult patients with suspected pulmonary mycobacterial disease.

The study population was comprised of consecutive patients admitted to the Dept of Pulmonary Diseases of Ambroise Paré University Hospital (Boulogne, France) between January 2003 and March 2009 because of a clinical suspicion of pulmonary mycobacterial disease. The study was approved by the Ethics Committee for the Protection of Subjects Participating in Biomedical Research at our institution. Patients were included in the study if a spontaneously expectorated sputum sample could not be obtained or was insufficient in volume. 